Research Paper

20184106

\title{
Model Reduction Technique for Analysing Friction-Induced Vibrations and Radiated Sound
}

\author{
Konrad Weisheit ${ }^{1)}$ Steffen Marburg ${ }^{1)}$ \\ 1) Technical University of Munich, Department of Mechanical Engineering, Chair of Vibroacoustics of Vehicles and Machines \\ Boltzmannstaße 15, 85748 Garching bei München, Germany (E-mail: Konrad.Weisheit@TUM.de)
}

Received on March 14, 2017

\begin{abstract}
A novel approach for the calculation of friction induced vibration is presented and applied to a real life example. The balance equations of continuum mechanics representing the underlying physics are simplified and then solved numerically. First, a linearised model of the example system is built to extract modal parameters such as eigenvalues and eigenvectors. A subset of eigenvectors is then used to calculate the system's response due to friction related excitation. This non-linear vibration is calculated by utilising an explicit time integration scheme. With the use of a subset of eigenvectors the degree of freedom of the system is reduced drastically, leading to considerable reduction of computational effort. The solution is compared to results from adequate experiments. Although developed in a context of noise calculation in the automotive sector, this work focuses on the calculation of friction induced vibration, rather than on noise prediction. Nevertheless, as an example the resulting noise of the vibrating system is estimated by means of the Equivalent Radiated Power (ERP).
\end{abstract}

KEY WORDS: Vibration, Noise and Ride Comfort, Accessory and Device Noise, Friction Induced Vibration, Equivalent Radiated Power, Experimental and Numerical Study, Modal Analysis [B3]

\section{Introduction}

Friction induced vibrations are present in many technical applications and in most cases are unwanted. On the one hand these vibrations can lead to harmful working conditions with system failure, on the other hand they can cause noise radiation and thus they reduce comfort. Examples for friction induced sound are creaking doors, squealing breaks, chalk on a blackboard or in a more pleasant way: music of the violin and other bowed string instruments. A high level of effort has been made in research areas dealing with friction induced vibration, e.g. studies on brake squeal are well represented in literature ${ }^{(1-9)}$.

In the automotive industry, besides brake squeal, squeaking noise in the passenger cabin is a major issue. This transient noise phenomenon appears in a random manner and reduces the passenger's comfort and therefore, a vehicles' quality rating. Especially in the segment of upper class vehicles, customers are not willing to accept squeak (and rattle) noise. This issue has become even more evident with the improvements in the fields of engine sound, wind noise and other acoustic relevant sectors.

For this reason, numerous tests are performed among the automotive companies to reduce squeak and rattle noise during characteristic drive situations. Due to the lack of advanced and reliable methods to predict squeak and rattle (SAR) noise, solutions are often settled in a late stage of product development. Finding these secondary solutions is exceptionally time and cost consuming ${ }^{(10-12)}$. Therefore, further investigations have to be accomplished in order to account for these phenomenas in an early stage of product development and thus reducing the cost of secondary measures. Since the field of SAR noise generation is not limited to the automotive sector, it is well studied and documented in the literature ${ }^{(13-19)}$

Numerical methods such as the Finite Element Method (FEM) are useful engineering tools to find critical spots in a vehicle's interior which can be subject to SAR phenomena. The advantage of these methods is, that they can be applied in an early stage of development and so help to reduce costs significantly. Well advanced for rattle problems the prediction of squeak noise is still subject to fundamental research. A new approach to calculate friction induced vibrations and the directly coupled radiated sound is presented in this work. By finding an acceptable estimation of the dynamic behaviour while reducing the number of degrees of freedom the novel method satisfies the compromise between solution quality and computational cost.

The outline is as follows: In section 2, the test rig SSP-03 by Ziegler Instruments ${ }^{(20)}$ is presented. It is used to evaluate the dynamic behaviour of material combinations which are subject to SAR noise phenomena when utilised in the interior of a vehicle. The test rig provides experimental data and serves as example problem for the developed method.

Section 3 presents the derivation of a numerical model making use of the FEM. Modal parameters such as modal mass, modal stiffness, eigenvalues and eigenvectors are extracted from a linearized model. Common methods of Finite Element theory ${ }^{(21-}$ 23) are used and modal reduction, which has efficiently been used in structural dynamics for decades ${ }^{(24-25)}$ is performed. This method follows the simple modal superposition of a subset of degrees of freedom ${ }^{(26)}$. The structural dynamics as well as the force due to 
friction related mechanisms are described in terms of modal coordinates $^{(27-28)}$.

The authors are aware that the friction force is not a given external force and that the eigenvalues depend on the frictional contact, even when the most simple friction law is applied. Like in linear dynamics the eigenvectors are used to transform the system of equations, but in contrast the equations of motion are still coupled when frictional effects are involved. The inherited character of the friction force is preserved. The approximation only consists in terms of modal reduction. This reformulation can be seen as preconditioning the system of equations which makes the computation more efficient when using an explicit time step integration scheme.

In section 4, the equations of motion are solved by using a Runge-Kutta scheme. The results are displacement, velocity and acceleration at specific locations. The numerical solutions are compared to experimentally gained data. Finally, the structural vibration, i.e. the normal surface velocity is used to evaluate the Equivalent Radiated Power (ERP) ${ }^{(29)}$.

In the last section, conclusions are drawn and recommendations for future work are proposed.

\section{The SSP-03 test rig}

The SSP-03 test rig is used to evaluate the dynamic behaviour and the contact physics of different material pairs as well as their tendency to produce squeak noise. Typical combinations of interest are: leather vs. leather, leather vs. polymer, polymer vs. polymer and rubber vs. metal. Figure 1 shows the experimental setup.

The materials to be tested are glued to the measuring adapter (C) and the measuring slide (D). After the two samples are brought into contact the measuring slide is moved in horizontal $y-$ direction. When motion begins the measuring adapter sticks to the measuring slide. The frictional force in the contact pair leads to a displacement of the adapter. With growing deflection, the restoring force exceeds the maximum value of the static friction force and sliding begins. Three different kinds of dynamic behaviour can be observed. The sliding can continue without reaching a sticking state again. That is, the system will either reach a state of equilibrium, where the restoring force matches the dynamic friction force and the velocity of the measuring adapter is zero. Or undamped vibrations without sticking phases can occur. In the third case, the measuring adapter reaches the stick state during cyclic motion again. Because of the difference between static and dynamic friction coefficient the equations of motion change abrupt in the transition of sticking and sliding. The latter two cases, especially the third one are critical with respect to squeak noise phenomena. While testing, acceleration and displacement of the measuring adapter are recorded. If a material pair tends to squeak, the displacement shows a characteristic saw tooth tread profile. The maximum acceleration is investigated to determine whether the risk of squeak is acceptable or not. All tests are performed applying different contact normal forces, measuring slide velocity and temperature. If the acceleration exceeds a certain value in one of those tests, the material pair is labeled a squeak risk.
In a different way from it's usual application, the SSP-03 is utilized as a realistic test case in this work, to validate the method presented herein.

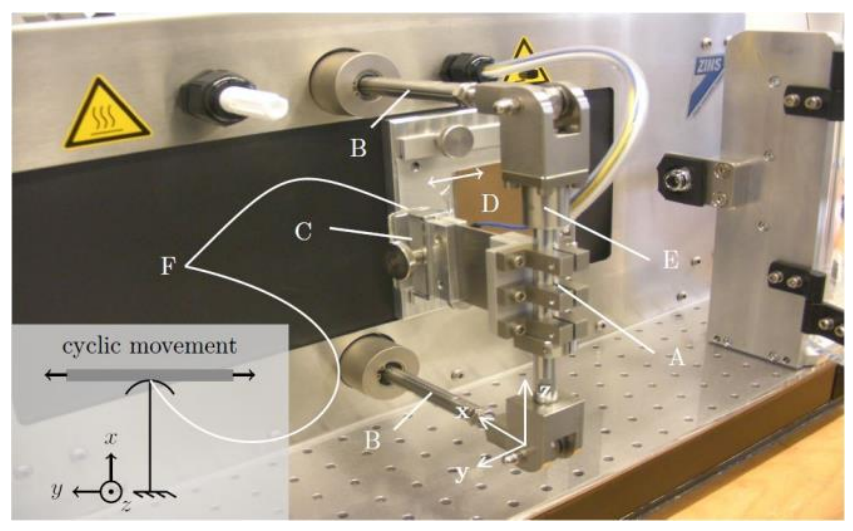

Fig. 1 Experimental setup of the SSP-03: A: framework, B: actuator rods, C: measuring adapter, D: measuring slide, E: linear roll bearing, F: contact area.

\section{Finite Element model and derivation of an analytical model}

\subsection{The Finite Element model of the configuration}

A Finite Element model has been developed to determine the vibration characteristics of the SSP-03. It consists of framework, measuring adapter and actuator rods, modeled with elastic 3D continuum elements. The remaining parts of the test rig (see Figure 1) are either assumed to be irrelevant to the investigation or to have a much higher stiffness. Thus, they are assumed to be rigid. This leads to the Finite Element model shown in Figure 2. Two elastic supports have been added to account for the dynamic behaviour of the actuator bearings, cf. Figure 2 .

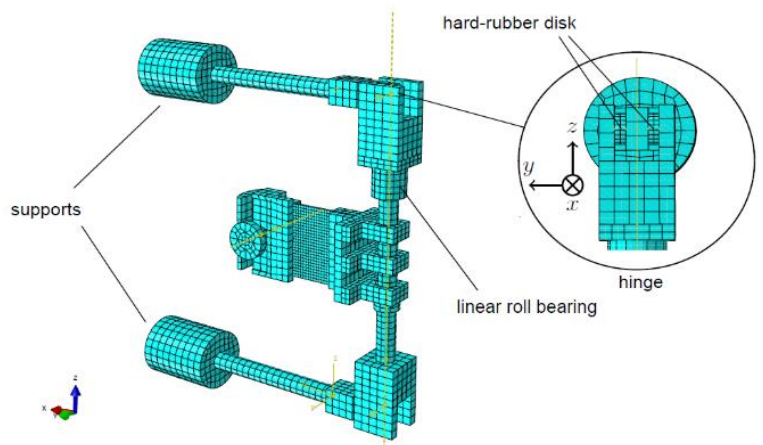

Fig. 2 Finite Element model of the configuration.

All screwed joints and the hinges are not represented in the model. Their influence is considered by applying appropriate kinematic constraints. This leads to some small visual deviations between the test rig shown in Figure 1 and the model in Figure 2.

The geometry and the material properties of the main components are well defined. In contrast, the dynamic behaviour of the supports and the hinges are uncertain and therefore need further investigation. To cope with this lack of information, an experimental modal analysis has been performed to find the eigenfrequencies of the setup. By comparing the eigenfrequencies of the numerical and the experimental modal analysis, the material properties of the support and the hinges are derived. The Young's- 
Moduli of these materials are set to such values, that the first three eigenfrequencies of the Finite Element model are in good agreement with those measured at the test rig.

After that, the Finite Element model is assumed to sufficiently represent the real test rig. It is used to calculate the eigenfrequencies and eigenvectors of the configuration in a frequency range up to $300 \mathrm{~Hz}$. Non-linearities are not accounted for. Figure 3 presents the results.

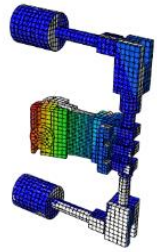

(a)

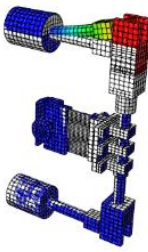

(d)

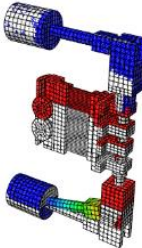

(b)

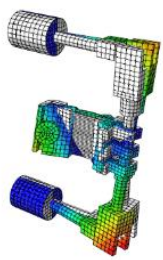

(e)

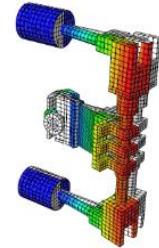

(c)

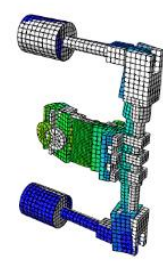

(f)
Fig. 3 Eigenfrequencies and eigenmodes at the SSP-03: (a) mode 1: $74 \mathrm{~Hz}$; (b) mode 2: $79 \mathrm{~Hz}$; (c) mode 3: $105 \mathrm{~Hz}$;

(d) mode 4: $130 \mathrm{~Hz}$; (e) mode 5: $183 \mathrm{~Hz}$; (f) mode 6: $274 \mathrm{~Hz}$.

The initial state is plotted in light grey color.

The modal analysis calculation delivers the information (eigenvectors, modal masses, modal stiffnesses) that is needed in the further calculation process, presented in the following section. They will be used to calculate the vibrations of the system, when a frictional force (applied by the measuring slide) is actuating on the measuring adapter. To keep these calculations cost effective, the subset of the first six eigenvectors is used in the later calculations. The advantage of using this modal represtentation rather than the direct approach, is that the degree of freedom is comparatively small, namely six.

It should be pointed out here, that the Finite Element model is built to find the modal parameters of the system. These values, along with the eigenvectors are needed in the analytical calculation presented later. They are used to perform a couple of transformations in a rather mathematical manner. However, they are not the modal parameters of the system and loose their physical significance once friction is applied.

The work flow is as follows: Starting from the actual physical configuration, assumptions for contact and boundary conditions are made to gain the mathematical model of the idealised system. A Finite Element model is built to approximate the mathematical problem. No non-linearities are taken into account, when the Finite Element model is used to determine the modal parameters of the system. These modal parameters are the input for the analytical solution, where the frictional contact now is considered and the vibrations of the system are calculated in time domain. With the velocity of the system's surface at hand, the Equivalent Radiated Power can be evaluated to estimate the radiated sound. Figure 4 shows the procedure.

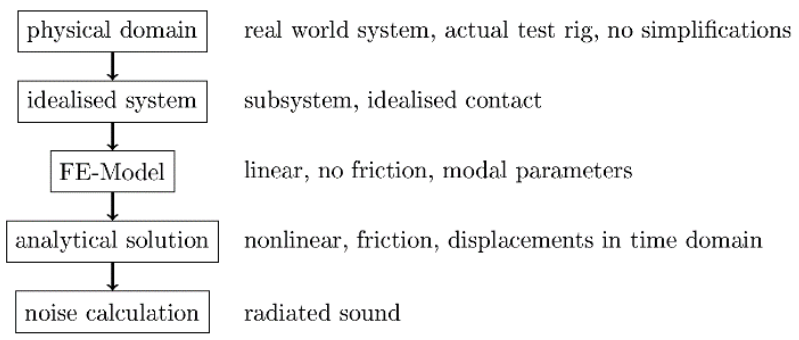

Fig. 4 Workflow of the noise calculation.

\subsection{Development of an analytical model}

The equation of motion for a linear system (no friction) of $n$ degrees of freedom without damping can be written as

$\mathbf{M} \ddot{\mathbf{q}}(t)+\mathbf{K q}(t)=\mathbf{f}(t)$

where $\mathbf{M}, \mathbf{K}, \mathbf{q}$ and $\mathbf{f}$ are the mass matrix, the stiffness matrix, the generalised coordinates and the vector of forces, acting on the system, respectively. In general, the equations in (1) are coupled. To find the solution, vector $\mathbf{q}$ can be expressed by modal coordinates $\xi$

$\mathbf{q}(t)=\sum_{j=1}^{n} \vartheta_{j} \zeta_{j}(t)=\boldsymbol{\Theta} \zeta(t)$.

The columns of the modal matrix $\boldsymbol{\Theta}$ are the eigenvectors $\vartheta_{j}$. The vector $\zeta$ consists of the modal coordinates. Inserting equation (2) in (1) and multiplying $\boldsymbol{\Theta}^{\mathrm{T}}$ from the left hand side leads to

$\boldsymbol{\Theta}^{\mathrm{T}} \mathbf{M} \boldsymbol{\Theta} \ddot{\zeta}(t)+\boldsymbol{\Theta}^{\mathrm{T}} \mathbf{K} \boldsymbol{\Theta} \zeta(t)=\boldsymbol{\Theta}^{\mathrm{T}} \mathbf{f}(t)$

As the eigenvectors are orthogonal, the matrices on the left hand side of equation (3) are diagonal, i.e.

$$
\begin{aligned}
& \boldsymbol{\Theta}^{\mathrm{T}} \mathbf{M} \boldsymbol{\Theta}=\tilde{\mathbf{M}}=\operatorname{diag}(\tilde{\mathbf{m}}), \\
& \boldsymbol{\Theta}^{\mathrm{T}} \mathbf{K} \boldsymbol{\Theta}=\tilde{\mathbf{K}}=\operatorname{diag}(\tilde{\mathbf{k}}),
\end{aligned}
$$

where $\tilde{\mathbf{m}}$ is a vector, containing the modal masses $\tilde{m}_{j}$, corresponding to the squared eigenfrequency $\omega_{j}^{2}$ and $\tilde{\mathbf{k}}$ consists of the modal stiffnesses $\tilde{k}_{j}$. The eigenvectors, modal masses and modal stiffnesses are known from the Finite Element calculation. Hence, all parameters on the left-hand side of equation (3) are identified. The right-hand side however depends on the vector of forces $\mathbf{f}(t)$ which act on the system. In the case at hand, this vector consists of frictional forces which lead to a coupling between the equations in (3).

For the analytical calculation presented later, it is desirable to take as few modes as possible into account, in order to keep the calculation cost effective. On the other hand, a minimum number of modes has to be used to achieve good quality results. In this 
work, all eigenvectors with a corresponding eigenfrequency below $300 \mathrm{~Hz}$ are chosen to represent the system, namely the six eigenvectors shown in Figure 3. In this case, the modal matrix $\boldsymbol{\Theta}$ is of size $6 \times 6$.

It should be pointed out again, that the projection (3) does not lead to a decoupling of the equations of motion, as the equations on the right hand side of this system still include terms of all modal coordinates in all lines. The projection is a sheer mathematical transformation that helps handling the system of equations of motion.

There are six degrees of freedom left and the system of equations (3) reduces to

$\tilde{m}_{j} \ddot{\zeta}_{j}(t)+\tilde{b}_{j} \dot{\zeta}_{j}(t)+\tilde{k}_{j} \zeta_{j}(t)=\vartheta_{j}^{T} \mathbf{f}(t)=\tilde{f}_{j}(t)$,

with $j=1, . ., 6$ and no summation over the index $j$. In equation (5), $\tilde{b}_{j}$ represent small viscous damping. There are no further external forces acting on the system, what leaves the friction force to be determined. Several friction laws can be defined ${ }^{(30-32)}$.

Herein, an exponential approach has been chosen to describe the friction coefficient

$\mu=\mu_{k}+\left(\mu_{s}-\mu_{k}\right) \cdot e^{-\gamma\left|v_{r e l}\right|}=\mu_{k}+{ }_{\Delta} \mu \cdot e^{-\gamma\left|v_{r e l}\right|}$

where $\mu_{s}$ and $\mu_{k}$ are the static and the kinetic friction coefficient, respectively. The parameter $\gamma$ describes the decay of the friction coefficient with the relative velocity $v_{r e l}$ between the contact partners. Figure 5 shows a typical graph of the friction coefficient.

Using this friction law and assuming that the friction force acts in $y$-direction only, the force acting on node $k$ in $y-$ direction can be determined by

$$
\begin{aligned}
& F_{R}^{k}(t)=F_{N}^{k}\left[\mu_{k}+{ }_{\Delta} \mu \cdot e^{-\gamma\left(v_{0}-v_{k}(t)\right)}\right] \\
& \forall v_{k}(t) \leq v_{0},
\end{aligned}
$$

or

$$
\begin{aligned}
& F_{R}^{k}(t)=-F_{N}^{k}\left[\mu_{k}+{ }_{\Delta} \mu \cdot e^{-\gamma\left(v_{k}(t)-v_{0}\right)}\right] \\
& \forall v_{k}(t)>v_{0},
\end{aligned}
$$

with the normal force $F_{N}^{k}$ at node $k$, the velocity of the measuring slide $v_{0}$ and the velocity of node $k$ in $y$-direction $v_{k}(t)$.

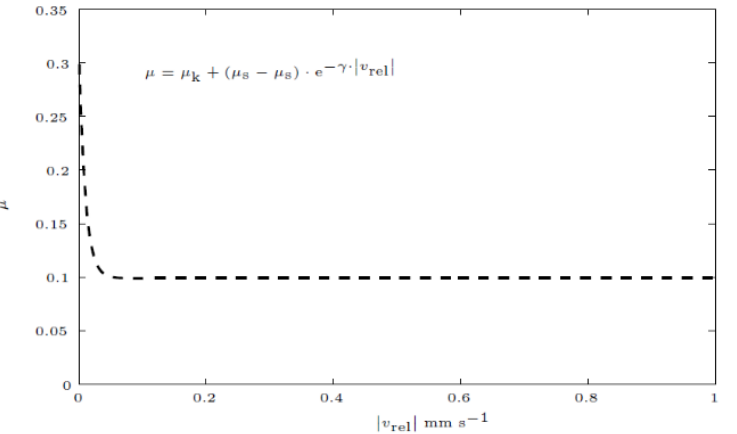

Fig. 5 Friction coefficient $\mu$ depending on the relative velocity.

Assuming that the friction force only acts on the nodes at the front of the measuring adapter (see Figure 6) and only in $y-$ direction, the right-hand side of equations (7) and (8) consists of a few terms only. Their number equals the number of nodes in contact

$\tilde{f}_{j}(t)=\sum_{k=1}^{n_{k}} \vartheta_{j}^{k} F_{N}^{k}\left(\mu_{k}+{ }_{\Delta} \mu \cdot e^{-\gamma\left(v_{0}-\sum_{i=1}^{6} \vartheta_{i}^{k} \dot{\zeta}_{i}(t)\right)}\right)$

$\forall v_{k}(t) \leq v_{0}$

$\tilde{f}_{j}(t)=-\sum_{k=1}^{n_{k}} \vartheta_{j}^{k} F_{N}^{k}\left(\mu_{k}+{ }_{\Delta} \mu \cdot e^{-\gamma\left(\sum_{i=1}^{6} \vartheta_{i}^{k} \dot{\zeta}_{i}(t)-v_{0}\right)}\right)$

$\forall v_{k}(t)>v_{0}$

with $j=1, . ., 6$. In the equations above, $\vartheta_{j}^{k}$ is the $y-$ component of eigenvector $\vartheta_{j}$ at node $k$. The velocity $v_{k}(t)$ in equations (7) and (8) has been replaced using

$$
\dot{\mathbf{q}}=\boldsymbol{\Theta} \dot{\zeta}
$$

Considering modal damping in equations (9) and (10), the equation of motion finally takes the form

$\ddot{\zeta}_{j}=\widetilde{m}_{j}^{-1}\left[-\tilde{k}_{j} \zeta_{j}-\tilde{b}_{j} \dot{\zeta}_{j}\right]+$

$\tilde{m}_{j}^{-1} \sum_{k=1}^{n_{k}} \vartheta_{j}^{k} F_{N}^{k}\left(\mu_{k}+{ }_{\Delta} \mu \cdot e^{-\gamma\left(v_{0}-\sum_{i=1}^{6} \vartheta_{i}^{k} \dot{\zeta}_{i}(t)\right)}\right)$

$\forall v_{k}(t) \leq v_{0}$

and

$\ddot{\zeta}_{j}=\tilde{m}_{j}^{-1}\left[-\tilde{k}_{j} \zeta_{j}-\tilde{b}_{j} \dot{\zeta}_{j}\right]-$

$\tilde{m}_{j}^{-1} \sum_{k=1}^{n_{k}} \vartheta_{j}^{k} F_{N}^{k}\left(\mu_{k}+{ }_{\Delta} \mu \cdot e^{-\gamma\left(\sum_{i=1}^{6} \vartheta_{i}^{k} \dot{\zeta}_{i}(t)-v_{0}\right)}\right)$

$\forall v_{k}(t)>v_{0}$

with $j=1, . ., 6$ and the modal damping $\tilde{b}_{j}$. It can easily be seen now, that the exponents in equations (12) and (13) lead to a coupling of the equations of motion. As mentioned before, this is usually the case in the presence of friction forces. 


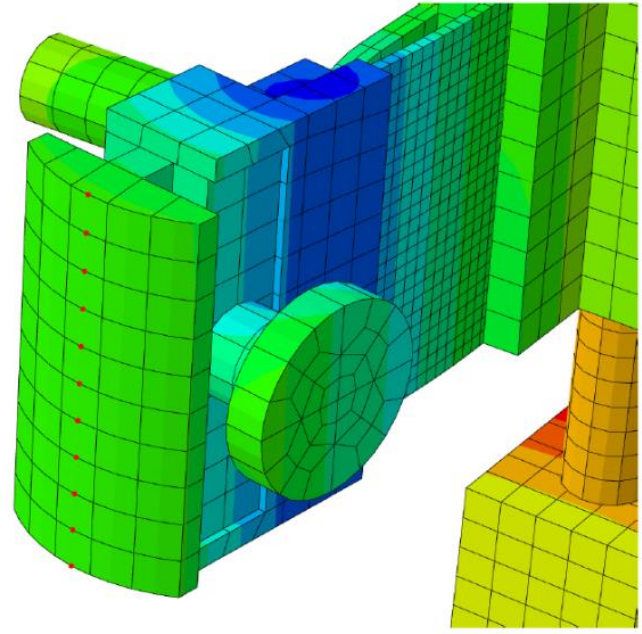

Fig. 6 Nodes in contact with the measuring slide (red dots).

\section{Simulation}

4.1. Integration of the equations of motion using the Runge-Kutta algorithm

The equations of motion in the previous chapter have the form $\ddot{x}=f(t, x, \dot{x})$.

With given initial conditions, these equations can be integrated numerically, using the Runge-Kutta scheme for example. The results are the velocities and displacements at discrete time steps in modal coordinates. The displacements and velocities in generalised coordinates are then obtained according to equations (2) and (11). Table 1 shows the modal parameters that were used.

Table 1 Parameters obtained using the Finite Element model.

\begin{tabular}{c|c|c|c|c}
$\begin{array}{c}\bmod \\
\mathrm{e}\end{array}$ & $f(\mathrm{~Hz})$ & $\tilde{m}_{j}(\mathrm{~kg})$ & $\tilde{k}_{j}\left(\mathrm{~kg} \mathrm{~s}^{-2}\right)$ & $\tilde{b}_{j}\left(\mathrm{~kg} \mathrm{~s}^{-1}\right)$ \\
\hline \hline 1 & 74 & 0.10 & 21809 & 1.9 \\
\hline 2 & 79 & 0.73 & 180673 & 13.8 \\
\hline 3 & 105 & 0.59 & 258329 & 15.9 \\
\hline 4 & 130 & 0.27 & 178091 & 9.2 \\
\hline 5 & 179 & 0.26 & 336606 & 14.9 \\
\hline 6 & 269 & 0.17 & 474343 & 16.8 \\
\hline
\end{tabular}

The damping parameters in Table 1 have been chosen such, that Lehr's Damping ratio $D_{j}\left(\tilde{b}_{j}=2 D_{j} m_{j} \omega_{j}\right)$ takes values between 0.0205 and 0.03 , corresponding to the results of the experimental modal analysis.

\subsection{Measurement as a reference for validation}

A measurement has been performed at the SSP-03 test rig. Artificial leather has been tested versus itself. A normal force of 5 $\mathrm{N}$ and a measuring slide velocity of $v_{0}=2 \mathrm{~mm} \mathrm{~s}^{-1}$ have been applied. The velocity of the measuring slide (D in Figure 1) was kept constant and did not change direction. The sampling rate was $1200 \mathrm{~Hz}$. While the accelerometer is located directly at the measuring adapter, the displacement (and it's time derivative, the velocity) are recorded at the spring between measuring adapter and framework.
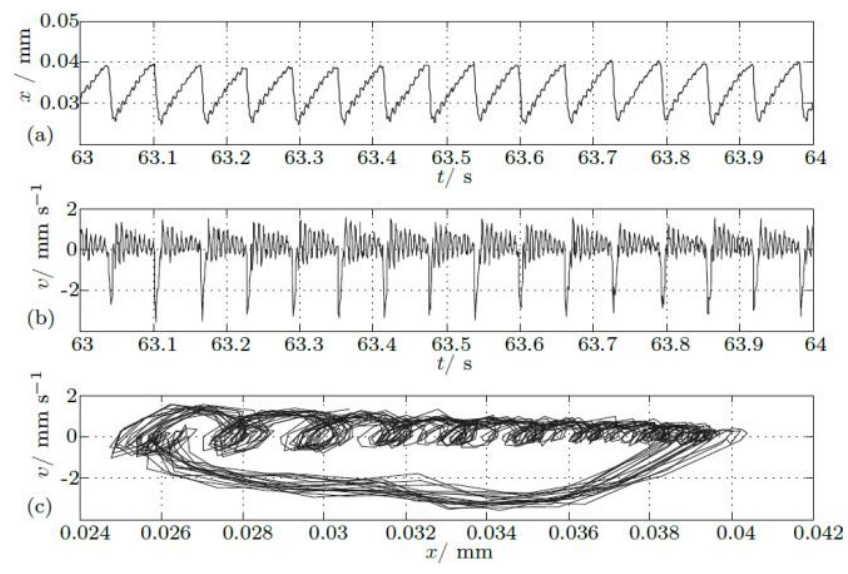

Fig. 7 Extraction of the signals at the test rig: (a) displacement over time, (b) velocity over time, (c) velocity over displacement.

Figure 7 shows an extraction from the measurement. The values of displacement and velocity are plotted over time. It can be observed that the velocity differs from that of the measuring slide and that vibrations are still present, even during the sticking phase. The reason is that the transducer for the displacement is located at the bending spring but not directly at the measuring adapter. While the measuring adapter sticks to the measuring slide and has the same velocity, the rest of the system keeps vibrating even during this sticking phase. Only in the contact area, these vibrations add up to the (constant) velocity $v_{0}$. At all the other locations, the velocity during the sticking period is not constant.

As expected, the displacement shows the typical saw-tooth profile. The velocity has it's largest value in the sliding phase. There are two peaks. One at the beginning of sliding, when the measuring adapter is released and one, with different sign, when sliding comes to an end and sticking starts.

The vibration shown in Figure 7 is the reference for the calculations following in the next section.

4.3. Determination of the frictional behavior from measurement results

Before calculations can be performed, the friction coefficients and the coefficient $\gamma$ have to be known. By calibration, the restoring force at the test rig can be determined from the deflection of the framework. Together with the preset normal force, this leads to the coefficients of friction $\mu_{s}$ and $\mu_{k}$. They are required for the calculation. In the test performed, the coefficients are determined to be

$\mu_{s}=0.68$,
$\mu_{k}=0.56$,

which is provided by the internal software of the test rig. However, it is possible to show, that the value of the kinetic friction coefficient is inaccurate, when determined in an experiment like that on the SSP-03 ${ }^{(33)}$. Together with the unknown coefficient $\gamma$ this means a major drawback when it comes to a validation of the presented method.

Nevertheless, the results for $\mu_{s}$ and $\mu_{k}$ have been used for a first attempt. The coefficient $\gamma$ in equation (6) was chosen to be 
$100 \mathrm{~s} \mathrm{~mm}^{-1}$, leading to an abrupt change between static and kinetic friction coefficient in the proximity of $v_{r e l}=0$. At $v_{r e l}=1 \mathrm{~mm} \mathrm{~s}^{-1}$ the difference between $\mu\left(v_{r e l}\right)$ and $\mu_{k}$ is less than $1 \%$.

With these information, a first set of calculations (A) is performed and shown in the next section.

\subsection{Calculation of motion and comparison to measurement}

With all the necessary information at hand, the equations shown in section 3 have been integrated numerically and resolved for displacement and velocity at various loacations at the system.

For a quality check, the movement at the contact area between measuring adapter and measuring slide is examined. As required, the velocity during the sticking phase is constant and has the same value as the measuring slide. In the phase of sliding, the velocity is below $v_{0}$ and changes sign. Thus, the results shown in Figure 8 are plausible.
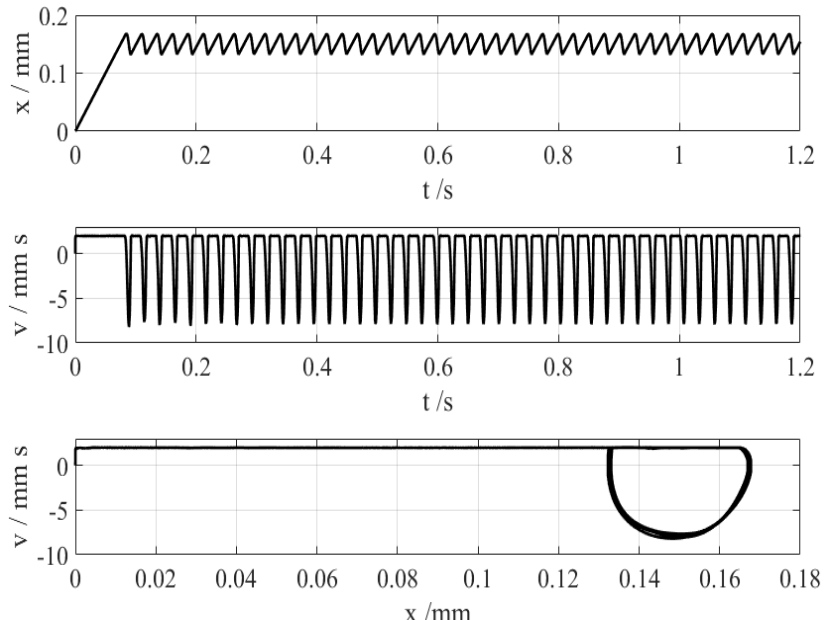

Fig. 8 Calculated displacement and velocity at the test rig; top: displacement over time, middle: velocity over time, bottom: velocity over displacement.

The movement in the contact area could not be recorded in the measurement. In order to compare the calculation with the experiment, the displacement at the transducer is investigated. It is presented in Figure 9. While the characteristics in general are similar to the behaviour in the test, some significant differences remain. The maximum displacement in the calculation is $0.037 \mathrm{~mm}$. While in the experiment it was $0.040 \mathrm{~mm}$. The sticking time $T_{H}$ is shorter and there are not as many oscillations during this phase as there were in the test. The shorter sticking period leads to a shorter period time as well. The calculation reaches 39 periods within one second, while in experiment, there were only 16 . The extreme values delivered by the calculation differ from those of the experiment as well. Table 2 shows the values of the experiment and the calculation (A). The table also shows the results of another calculation (B), where different coefficients of friction have been used.

The real frictional behaviour of the testet material pair is unknown. In order to achieve a better accordance between calculation an measurement, the coefficients of friction used in the calculation have been changed in the following manner. The coefficient of static friction has been increased, so that the maximum displacement in the calculation is $0.040 \mathrm{~mm}$, the same as it was in the experiment. On the other hand, the coefficient of kinetic friction has been decreased, making the measuring adapter sliding farther back before it sticks to the measuring plate again. This leads to an increased sticking time and consequently to an increased period time.

With the two adjustments, the maximum deflection at the location of the transducer and the period time $T_{0}$ of the vibration match between calculation and measurement. This should also lead to corresponding "frequency content" of the both vibrations.

The second calculation was performed with $\mu_{s}=0.74$ and $\mu_{k}=0.47$. All the other parameters remain unchanged. Figure 10 shows the results.
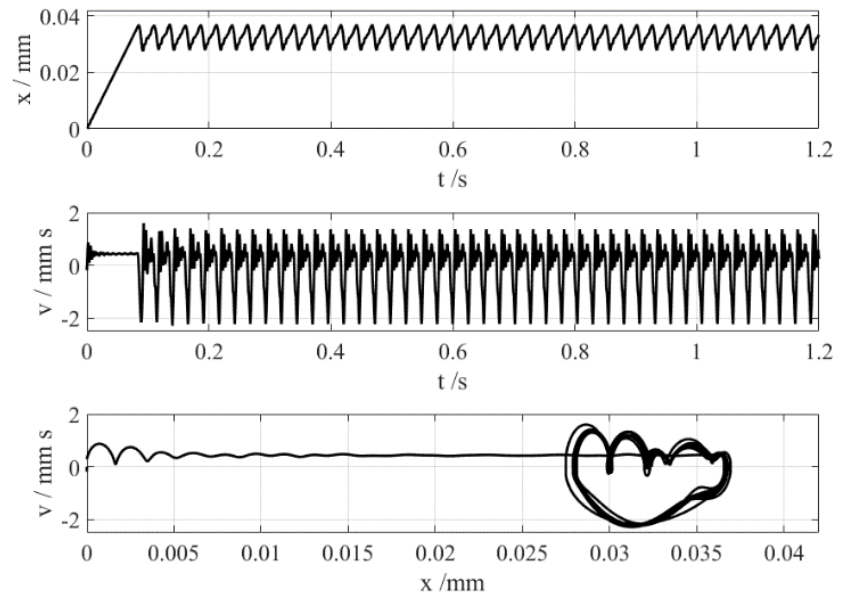

Fig. 9 Displacement and velocity at the position of the transducer (calculation A); top: displacement over time, middle: velocity over time, bottom: velocity over displacement.

While the time periods in calculation $B$ match those of the experiment now, the differences for $y_{\min }, v_{\min }$ and $v_{\max }$ have become larger.

Table 2 Comparison between experiment and calculation.

\begin{tabular}{c|c|c|c} 
& Exp. & Calc. A & Calc B \\
\hline$y_{\max }(\mathrm{mm})$ & 0.040 & 0.037 & 0.040 \\
\hline$y_{\min }(\mathrm{mm})$ & 0.025 & 0.028 & 0.012 \\
\hline$v_{\max }\left(\mathrm{mm} \mathrm{s}^{-1}\right)$ & 1.5 & 1.4 & 3.8 \\
\hline$v_{\min }\left(\mathrm{mm} \mathrm{s}^{-1}\right)$ & -4.0 & -2.2 & -6.4 \\
\hline$T_{0}(\mathrm{~s})$ & 0.06 & 0.03 & 0.06 \\
\hline$T_{H}(\mathrm{~s})$ & 0.05 & 0.02 & 0.05 \\
\hline & & &
\end{tabular}

There are some possible explanations for the remaining differences between calculation and experiment. First of all, without extensive testing the friction law remains unknown. But, as the two calculations show, the friction law substantially influences the vibrational behaviour. With a validated friction law, a better agreement between calculation and experiment should be obtained. Furthermore, the system has been idealised. It has been assumed that the contact area between measuring adapter and measuring slide is a straight line, as shown in Figure 6. In many 
tests, this is not the case and only the upper end of that line is in contact. In general, an uneven distribution of the frictional load between the nodes shown in Figure 6 must be expected. Consequently, the spring will experience a torsional load in addition to the bending load applied here.

While there are differences between calculation and experiment in some values, the overall behaviour of the system is represented quite realistic. There are phases of sticking and sliding in the contact area, the velocity during sticking is $v_{0}$. In some distance from the contact area, the system keeps vibrating during the sticking phase as was observed at the test rig.

In the next section, the results of the calculation are used to estimate the noise radiated by the system.
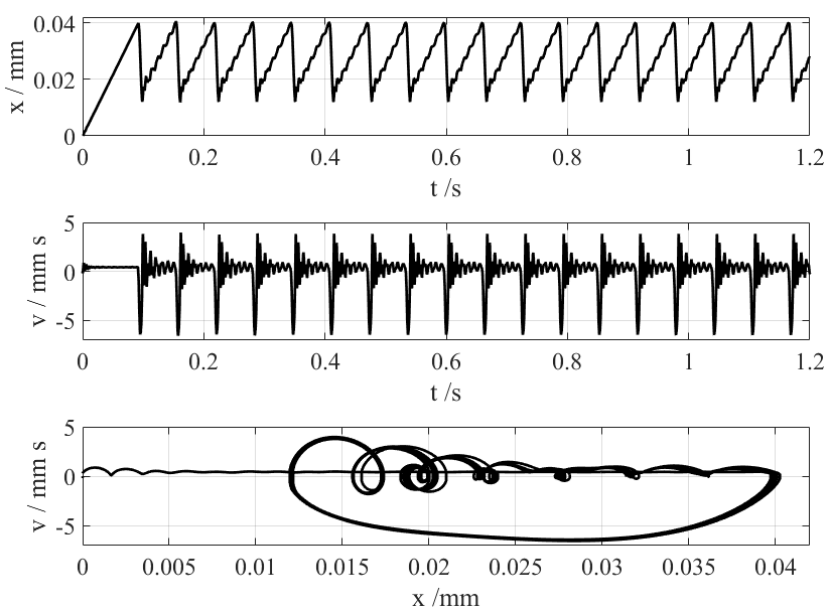

Fig. 10 Displacement and velocity at the position of the transducer (calculation B); top: displacement over time, middle: velocity over time, bottom: velocity over displacement.

\section{Estimation of the radiated sound by calculating the Eqivalent Radiated Power (ERP)}

The Equivalent Radiated Power is an estimate for the sound, radiated by a vibrating elastic body ${ }^{(28)}$. It is given by the following surface integral

$$
P_{E R P}=\frac{1}{2} \rho_{f} c_{f} \int|\mathbf{v}(\mathbf{x})|^{2} d O(\mathbf{x})
$$

and provides the radiated sound power under the assumption of a radiation efficiency of 1 . In (16), $\rho_{f}$ is the density of the surrounding fluid (e.g. air) and $c_{f}$ is the speed of sound in this fluid. Vector $\mathbf{v}(\mathbf{x})$ is the velocity at the body's surface in normal direction.

After applying the Finite Element Method, the surface of the system is discretized. Assuming the normal velocity does not vary over the small area of each surface element, the integral in (19) can be replaced by the sum

$$
P_{E R P} \approx \frac{1}{2} \rho_{f} c_{f} \sum_{k=1}^{N_{e}} A_{k}|\mathbf{v}(\mathbf{x})|^{2}
$$

with the number of surface elements $N_{e}$ and element area $A_{k}$.
In order to keep the computational effort low, only the faces relevant for the radiation of noise during stick-slip vibration are considered, i.e. faces at the measuring adapter, the bending spring and the framework.

From the calculation in section 4.2, the velocities in modal coordinates are known. In global coordinates they can be obtained according to equation (11). The result is transformed to the frequency domain by Fourier Transformation. Figure 11 shows the result for calculation B from section 4.2.

The signal consists of numerous equally spaced peaks. The lowest peak appears at $16 \mathrm{~Hz}$. This correlates to the period time of the stick-slip motion. The other peaks follow in intervals of $16 \mathrm{~Hz}$ and are resonances of the first harmonic. The sound signal in Figure 11 does not show any peaks at the eigenfrequencies $74 \mathrm{~Hz}$ and $105 \mathrm{~Hz}$, i.e. the sound is completely dominated by the stick-slip process, the free vibrations of the system during sticking remaining do hardly contribute to the squeak noise.

This result is interesting insofar that the resulting squeaking sound is not spoiled by the test rig construction. It can be assumed that this has been aimed at by the producer. Further investigation will show whether this feature is also observed for other materials.

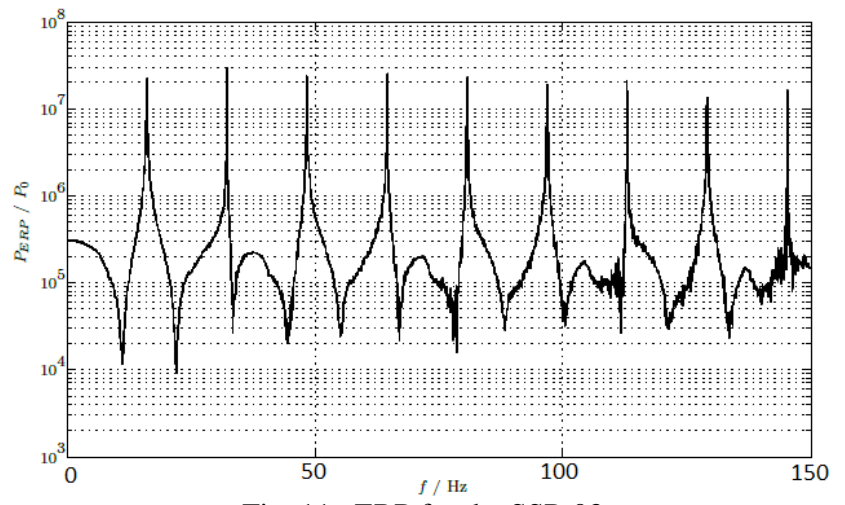

Fig. 11 ERP for the SSP-03.

\section{Conclusion}

In this work, an approach to calculate noise produced by friction induced vibrations is presented. It uses a simple modal reduction and explicit time integration. The system's motion is described in modal coordinates, taking a limited number of degrees of freedom into account. The friction force is modeled as a non-conservative actuator, which depends on the kinematics (velocities) of the system itself.

The method is applied to a test rig that is used to investigate materials regarding their suitability to be used in a car's interior. It is shown, that the friction law has great influence on the solutions quality.

The interesting feature of the new method is the calculation of the system's movement with only a few degrees of freedom and with much less effort, than the established methods might take. Both, modal analysis and time integration are very efficient and a suitable calculation time for the overall process can be achieved. The main objective was the calculation of friction induced vibration, especially the velocity on the systems surface.

The results were then used to calculate the Equivalent Radiated Power emitted by the stick-slip motion at the test rig. The information about the radiated noise is valuable to engineers developing a silent car interior, for example.

Before the method can be used in a development process, it should be expanded to at least two elastic bodies in contact and the option of external excitation. Furthermore, the validation should be extended about hardware tests with material pairs, for which the 
frictional behaviour is known in detail. As the friction law has great influence on the results, the quality of the new method can only be evaluated reliably with such knowledge. After all, the method presented here is a promising approach to calculate large scale systems in acceptable time to achieve good quality results.

This work was supported by the German Research Foundation (DFG) and the Technical University of Munich (TUM) in the framework of the Open Access Publishing Program.

\section{References}

(1) AbuBakar A. R. Ouyang H. : Complex eigenvalue analysis and dynamic transient analysis in predicting disk break squeal, Int. J. Vehicle Noise and Vibration, Vol. 2, p.143-155 (2006).

(2) Hetzler H. : On the effect of non-smooth Coulomb damping on flutter-type self-excitation in a non-gyroscopic circulatory 2DoF-system, Nonlinear Dynamics, Vol 73 Issue 3, p.1829-1847 (2013).

(3) Oberst S. Lai J. Marburg S. : Guidelines for numerical vibration and acoustic analysis of disk break squeal unsing simple models of break systems, Journal of Sound and Vibration, Vol. 332, p.2284-2289 (2013).

(4) Sinou J.-J. Thouverez F. Jezequel L. : Stability analysis and non-linear behaviour of structural systems using the complex nonlinear modal analysis (CNLMA), Computers \& Structures, Vol. 84, p.1891-1905 (2006).

(5) Coudeyras N. Nacivet S. Sinou J.-J. : Periodic and quasiperiodic solutions for multi-instabilities involved in break squeal, Journal of Sound and Vibration, Vol. 328, p.520-540 (2009).

(6) Duffour P. Woodhouse J. : Instability of systems with frictional point contact, part 1: basic modeling, Journal of Sound and Vibration, Vol. 271, p.365-390 (2004).

(7) Duffour P. Woodhouse J. : Instability of systems with frictional point contact, part 2: model extensions, Journal of Sound and Vibration, Vol. 271, p.391-410 (2004).

(8) Duffour P. Woodhouse J. : Instability of systems with frictional point contact, part 3: experimental tests, Journal of Sound and Vibration, Vol. 304, p.186-200 (2007).

(9) Butlin T. Woodhouse J. : Sensitivity of friction-induced vibration in idealized systems, Journal of Sound and Vibration, Vol. 319, p.182-198 (2009)

(10) Kavarana F. Rediers B. : Squeak and rattle - state of the art and beyond, Sound and Vibration (2001).

(11) Kreppold E. : A modern development process to bring silence into interior components, SAE Technical Paper, No. 200701-1219 (2007).

(12) Moosmayr T. : Objektivierung von transienten Störgeräuschen im Fahrzeuginnenraum (objectivity of transient vehicle noises), Ph.D. Thesis, TU Munich, Munich, Germany (2008).

(13) Naganarayana B. Shankar S. Bhattachar V. Brines R.S. Rao S.R. : Nhance: software for identification of critical bsr locations in automotive assemblies using finite element models, SAE paper, No. 2003-01-1522 (2003).

(14) Nolan S.A. Loftus L. : Vehicle squeak and rattle benchmarking, IMAC IVX, SEM, p. 483-489 (1996).

(15) Nolan S.A. Smut J. : Automotive squeak and rattle prevention, SAE paper, No. 921065 (1992).

(16) Nolan S.A. Yao Y. Tran V. Weber W. Heard G. : Instrument panel squeak and rattle testing and requirements, IMAC IVX, SEM, p. 490-494 (1996).

(17) Rorrer R.A. Juneja V. : Friction induced vibration and noise generation of instrument panel material pairs, Tribology International, Vol. 35, p.523-531 (2002).
(18) Sohmshetty R. Kappagantu R. Naganarayana B. Shankar S. : Automotive body structure enhancement for buzz, squeak rattle, SAE Technical Paper, No. 2004-01-0388 (2004).

(19) Weisheit K. Marburg S. : Calculation of the response of a periodically excited beam with frictional contact using Harmonic Balance Method, Procedia IUTAM, Vol 19, p.282-288 (2016).

(20) Ziegler : SSP-03, URL https://www.zins-zieglerinstruments.com/en/, (Accessed. September. 2018).

(21) Bathe K.-J. : Finite Element procedures, Prentice Hall (2006).

(22) Belytschko T. : Nonlinear Finite Elements for continua and structures, John Wiley and Sons, (2000).

(23) Smith M. : ABAQUS/Standard User's Manual Version 6.9, Simulia, (2009).

(24) Rayleigh J. W. S. : The theory of sound, Dover Publications New York (1945).

(25) Rixen D. J. Geradin M. : Mechanical vibrations: theory and application to structural dynamics, John Wiley and Sons, Vol. 2, (1997).

(26) Craig R. R. Kurdila A. J. : Fundamentals of structural dynamics, John Wiley and Sons Hoboken, Vol. 2, (2006).

(27) Dresig H. Holzweißig F. : Dynamics of machinery, Springer Heidelberg Dordrecht London New York, Vol. 12, (2010).

(28) Magnus K. Popp K. : Schwingungen (Oscillations), Teubner Wiesbaden, Vol. 7, (2005).

(29) Fritze D. Marburg S. Hardtke H.-J. : Estimation of radiated sound power: a case study on common approximation methods, Acta Acustica united with Acustica, Vol. 95, p.833-842 (2009).

(30) Persson B. N. : Sliding friction, Springer Berlin Heidelberg New York, Vol. 2, (2000).

(31) Popov V. : Contact mechanics and friction, Springer

Heidelberg Dordrecht London New York, (2010).

(32) Rabinowicz E. : Friction and wear of materials, Wiley, Vol. 2, (1995).

(33) Oden J.T. Martins J.A.C. : Models and computational methods for dynamic friction phenomena, Computer Methods in Applied Mechanics and Engineering, Vol. 52, p. 527-634 (1985). 